\title{
Training for the 1st international nano-car race: the Dresden molecule-vehicle
}

\author{
Frank Eisenhut ${ }^{1}$, Corentin Durand ${ }^{2}$, Francesca Moresco $^{1}$, Jean-Pierre Launay ${ }^{2}$, and Christian Joachim ${ }^{2,3, a}$ \\ ${ }^{1}$ Institute for Materials Science, Max Bergmann Center of Biomaterials, and Center for Advancing Electronics Dresden, \\ TU Dresden, 01062 Dresden, Germany \\ 2 GNS \& MANA Satellite, CEMES, CNRS, 29 rue J. Marvig, 31055 Toulouse Cedex, France \\ 3 International Center for Materials Nanoarchitectonics (MANA), National Institute for Materials Science (NIMS), \\ 1-1 Namiki, Tsukuba, Ibaraki 305-0044, Japan
}

Received: 1 July 2016 / Received in final form: 4 October 2016 / Accepted: 6 October 2016

(c) EDP Sciences 2016

\begin{abstract}
The first international nano-car race will be held in Toulouse, France in spring 2017, with the participation of six international teams. The training session of the Dresden Team is reported using the Toulouse LT-UHV 4-STM reconfigured with four independent controllers in preparation for the race. During the training, the total $\mathrm{Au}(111)$ surface was prepared over the full gold substrate and a $90 \mathrm{~nm}$ long race track with two turns was defined according to the new nano-car race rules. The Dresden windmill molecule-vehicle was deposited in ultrahigh vacuum conditions, imaged, and manipulated by any one of the four tips on race tracks to reach a $5 \mathrm{~nm}$ per hour driving speed including the STM image time after a given driving bias voltage pulse. During the manipulation with one of the tips, it was possible to image with another tip in parallel. Strategies for a safe and fast driving was established by the Dresden team along the fcc rafter of the $\mathrm{Au}(111)$ surface together with the possibility to repair on the spot the destroyed molecule for example during the negotiation of a turn. Other teams will beneficiate from this experience to also improve their driving strategy.
\end{abstract}

\section{Introduction}

The first international race of molecule-vehicles (nanocars) will be held in Toulouse, France in spring 2017, as first announced at the beginning of 2013 [1]. This race at the nanoscale will bring together teams with different molecule-vehicles on a metallic surface (some with wheels and some without, but with wings or paddles). Each molecule-vehicle is to be driven by an inelastic electronic tunneling current phenomenon, using the tip of a scanning tunneling microscope (STM) at low temperature (LT) and in an ultrahigh vacuum (UHV) environment [2-4]. Four nano-cars can be driven in parallel, independently and on the same surface, thanks to the newly installed LT-UHV 4-STM instrument in Toulouse, built by ScientaOmicron and having four STM heads able to operate on the same surface [5].

The design, synthesis and driving attempts of molecule-vehicles have already a long history, starting in the middle end of the 90s $[6,7]$. The first complete designs and chemical synthesis of such molecules appears soon after this period, as recalled in [1]. Some moleculevehicles have a chassis, two to four wheels and a motor [8]

\footnotetext{
a e-mail: joachim@cemes.fr
}

or paddles [9,10]. All that equipment is covalently bound together to form a single molecule even if in certain cases the wheels are only weakly bonded to the chassis to favor rotation [11]. For those molecule-cars, the motor (or the paddles) is a truly distinguishable part of the molecular structure. Its mechanics are generally moving spatially in a semi-classical manner. Other molecule-vehicles have a chassis, no wheel nor apparent motor but wings ([12], see for example the organic parts in [13]). The motive power of this second class of nanoscale vehicles is relying on their peculiar low lying electronic excited states potential energy surface manifold, when adsorbed on a metallic surface [12]. They can be considered as equipped with a quantum motor [4]. The design, the optimization of semi-classical and quantum motors, and especially the understanding of the physics of their energy feeding and efficiency is one of the scientific interests of organizing the nanoscale race [14].

The first ever "macroscopic" automobile race have been organized by "Le Petit Journal" in July 1894 between Paris and Rouen (France) [15]. At that time, 102 teams registered, 21 effectively started the $126 \mathrm{~km}$ long race and 17 arrived in Rouen about $7 \mathrm{~h}$ later. In 1894, the car structures and propulsion modes were as diverse as the molecule-vehicles of the nano-race: $3-6$ wheels and 
steam, petrol, hydraulic, air compressor, gravity, gasoline and pedals propulsion.

To register for the first edition of the Toulouse nano-car race, a team had to deliver to the organizers before May 2016: (a) the detail of its institution (Academic, public, private), (b) the design of its molecule-vehicle including the delivery of the $x y z$ file coordinates of the corresponding atomic structure, (c) the propulsion mode, by inelastic tunneling effects, (d) the deposition conditions of the molecule-vehicles on an $\mathrm{Au}(111)$ surface, (e) if possible a first UHV-STM image of the molecule-vehicle and (f) the name and nationality of the LT-UHV-STM driver. That information was used for selecting the six teams accepted to race. Training sessions have been organized to learn the driving conditions on the LT-UHV 4-STM, which will be the workhorse for the competition [5]. For the nano-race in spring 2017, nine teams over the world have expressed their interest to participate, one team gave up at the end of 2015 and six were finally selected by the organizers in May 2016. A better success rate at the arrival than for the first macroscopic race between Paris and Rouen is expected. Among the six teams accepted, three moleculevehicles are equipped with molecular wheels, three have no wheels but a pair of paddles or 2-4 wings [16]. All details about the race, its organization, the sponsors and the officially registered teams can be found on its technical website [16].

Accepted early to run for the nano-race, the Dresden team with its four wings molecule-vehicle was the first team to be trained in Toulouse on the LT-UHV 4-STM. After respecting the six conditions listed above to register, the initial part of the Dresden training was to adapt the sublimation conditions of their molecule-vehicle to the specific LT-UHV 4-STM preparation chamber, to image by LT-UHV-STM their molecule-vehicle using any of the four tips, to learn in detail how to use the LT-UHV 4-STM control software, to select a good track on the $\mathrm{Au}(111)$ surface chosen for the race and to clean it. Following (c) i.e., without mechanically manipulating their moleculevehicle, the single molecule STM manipulation conditions obtained by the Dresden team on their Createc LT-UHV single tip STM instrument had also to be reproduced during the training on the Toulouse LT-UHV 4-STM because the coaxial wiring interconnecting the tips on the LT-UHV 4-STM is not identical to the Createc wiring of a single tip. Then, the real training for racing started in April 2016, with the aim of finding a good strategy to drive the Dresden molecule-vehicle along a pre-determined $\mathrm{Au}(111)$ track and respecting the race rules [16].

The Dresden team training session had led to the evaluation of the maximum speed that their moleculevehicle can run, how to pass a turn of the herringbone reconstruction on the $\mathrm{Au}(111)$ surface along the predetermined track and finally in case of an accident, how to reconstruct the molecule-vehicle on the spot. All those training sections are described step by step in this paper with the objective to help the other teams accepted for the nano-race to continue the optimization of their own molecule-vehicle and to learn the driving conditions on the peculiar $\mathrm{Au}(111)$ herringbone surface reconstruction.
This is an important point for the success of the competition since the race itself will only last not more than $38 \mathrm{~h}$ non-stop in two days and one night [15]. There will be no time for training in parallel to the competition.

In this paper, we present in Section 2 the preparation of the $\mathrm{Au}(111)$ surface for the nano-race and define the characteristics of a track along the herringbone reconstruction. In Section 3, we describe the Dresden windmill molecule-vehicle, its STM image characteristics and its controllability by inelastic electronic tunneling effects. Section 4 reports what the Dresden team had learnt about driving its windmill vehicle along a herringbone track and about its driving strategy, in particular to turn along a given $\mathrm{Au}(111)$ fcc track. Section 5 details some of the nano-car accidents which have occurred during the training and how the Dresden team succeeded to repair on the spot a few of the windmill vehicles to continue the run.

\section{Preparation of the tracks on the $A u\left(\begin{array}{lll}1 & 1 & 1\end{array}\right)$ surface}

The race tracks are prepared on a given small portion of the $\mathrm{Au}(111)$ surface. The sample is an $8 \mathrm{~mm}$ bulk gold pastille about $1.8 \mathrm{~mm}$ thick. This pastille will be maintained at a low temperature of $4.3 \mathrm{~K}$ and in UHV during the competition. The $\mathrm{Au}(111)$ surface has been selected because of its reconstruction forming the well known native herringbone patterns [17] and because all the registered molecule-vehicles and even the planar ones with no wheels will be physisorbed on this surface (see for example [12]). The gold pastille is a commercial single $\mathrm{Au}(111)$ crystal from MaTecK with only one of its two sides polished. After its introduction into the UHV preparation chamber of the Toulouse LT-UHV 4-STM chamber that has a base pressure of $1 \times 10^{-10}$ mbar, the surface is cleaned by $\mathrm{Ar}^{+}$sputtering at a pressure of $1 \times 10^{-5} \mathrm{mbar}$ and an energy of $1 \mathrm{keV}$ leading to a current of $5 \mu \mathrm{A}$ for $30 \mathrm{~min}$. Afterwards, the $\mathrm{Au}(111)$ sample was annealed to $500{ }^{\circ} \mathrm{C}$ for $20 \mathrm{~min}$. After four cycles of sputtering and annealing, an atomically clean $\mathrm{Au}(111)$ surface is obtained. The $\mathrm{Au}(111)$ surface terraces and mono atomic steps can be imaged by each scanner of the LT-UHV 4-STM together with the herringbone reconstruction as presented in Figure 1. Those images were recorded using all of the four piezo scanners of the LT-UHV 4-STM alternatively or in parallel. Special attention was drawn to the fact that not only the center of the gold surface must be atomically clean but also its total periphery, because each team will have to race on an area of the surface quadrant located at this periphery, about $0.6 \mathrm{~mm}$ away from the border of the pastille (Fig. 1 is indicative of the a-d possible quadrants location). This is due to the constrain that, for the competition, the STM tips must be mounted perpendicular to the tip holder (and the surface) to recover the same molecular manipulation conditions on the LT-UHV 4-STM instrument than on a single tip LT-UHV-STM [5]. This is imposing that the scanning and 

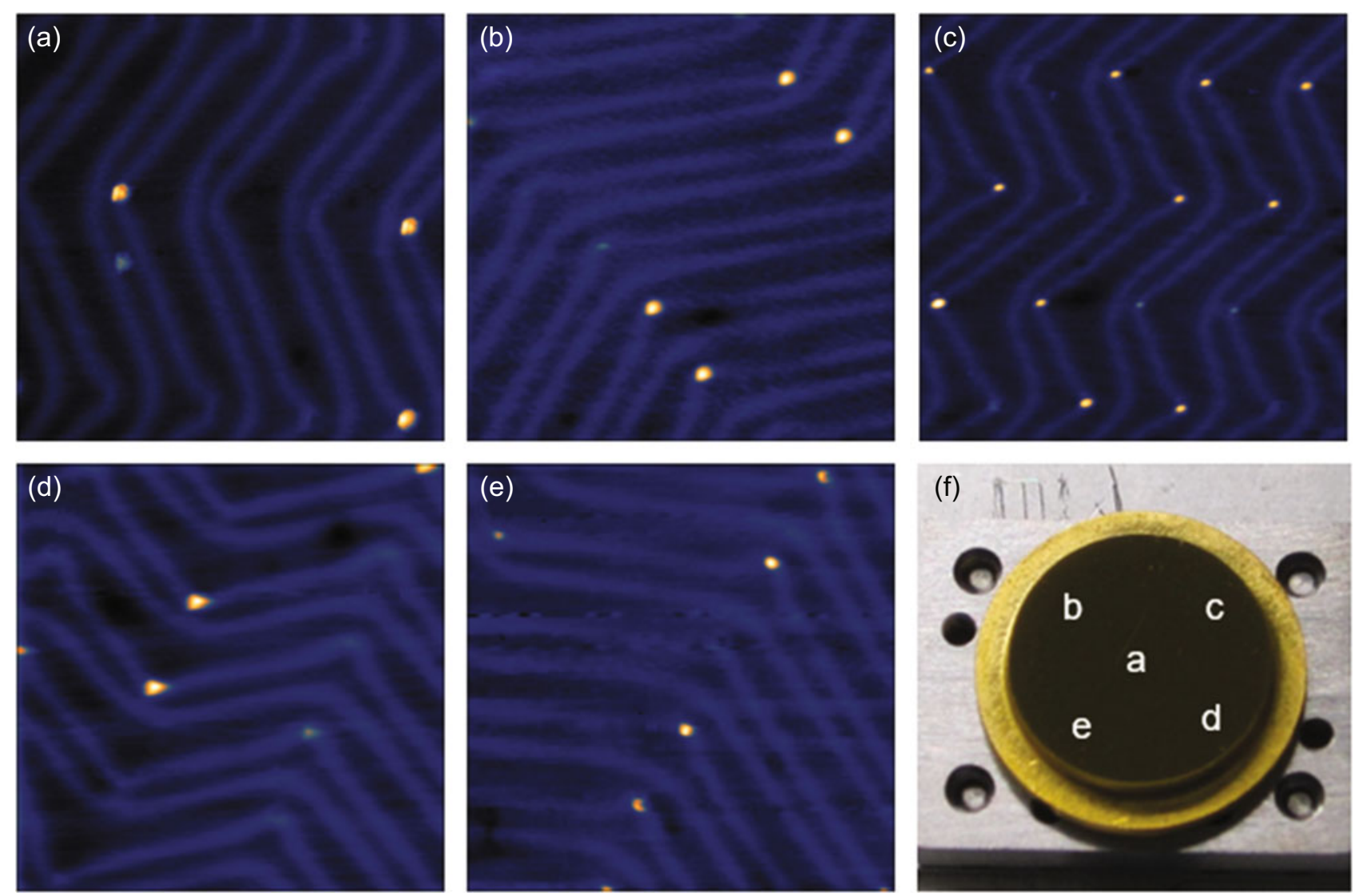

Fig. 1. LT-UHV constant current STM images of the prepared Au(111) surface for the Dresden training recorded with (a) scanner 4, (b) scanner 1, (c) again scanner 4, (d) scanner 3 and (e) scanner 2 on the Toulouse LT-UHV 4-STM instrument (image size: $40 \mathrm{~nm} \times 40 \mathrm{~nm} ; I=1.0 \mathrm{nA} ; V=1000 \mathrm{mV}$ ) and (f) an optical photo of the Au(111) pastille $8 \mathrm{~mm}$ in diameter mounted on its UHV sample holder with the STM image locations indicated for (a) to (e). Note the peculiar herringbone patterns with alternately narrow and wide zig-zag tracks separated by reconstruction walls acting as sliders (see text for discussion).

racing zone per team be located at the periphery of the $\mathrm{Au}(111)$ surface. Special elongated tip holders have been designed by the organizers to bring each tip in a vertical configuration as close as possible to the surface center with no change of resolution in the LT-UHV 4-STM.

During the Dresden training session, it was also certified that a well prepared gold pastille as presented in Figure 1 can travel back and forth many times between the preparation chamber and the 4 STM head of the LT-UHV 4-STM. There are four stops to mark in UHV along the path from the preparation-sublimation chamber and the storage carrousel of the 4-STM head for a travelling distance in UHV of about $2 \mathrm{~m}$. This was an important test for the competition since the molecule-vehicles of a given team will be evaporated and immediately STMimaged before the molecule-vehicles of the next team is deposited on the same surface. This will require at least 5 UHV transfers along the complete UHV chamber of the LT-UHV 4-STM to have all the teams ready for the nanorace. This UHV transfer sequence was successfully tested for the Dresden case since the on-surface synthesis dosing of the Dresden molecule-vehicles had required four back and forth UHV transfers to finally image the Dresden windmill molecules with a submonolayer coverage on the $\mathrm{Au}(111)$ surface (see Sect. 3 for more details).

When successfully prepared as presented in Figure 1, the $\mathrm{Au}(111)$ surface presents a typical herringbone pattern with the rafter corrugation of about $0.02 \mathrm{~nm}$. This defines an alternation of hcp and fcc domains (tracks) separated by the higher $0.02 \mathrm{~nm} \mathrm{Au}$ atomic rows which will constitute the sliders of the race track. The lateral width of an hcp region is about $2.5 \mathrm{~nm}$ and about $4.5 \mathrm{~nm}$ for the fcc from slider to slider. An fcc domain in between two sliders is ideal for a race track. It is wide enough to accommodate all the accepted molecule-vehicles and narrow enough to help in guiding them with the sliders to avoid any large and random lateral diffusion at low temperature. Additionally, some impurities at the elbows have been found, which came possibly from the back and forth transfer between preparation chamber and cryostat. The teams must take into account that for the race these defects must be avoided, as they can be seen as a barrier that the nano-cars cannot pass.

Initially, the nano-race organizers proposed the construction of an atomic scale slalom on the fcc domain. This would require the STM-manipulation of a few single 


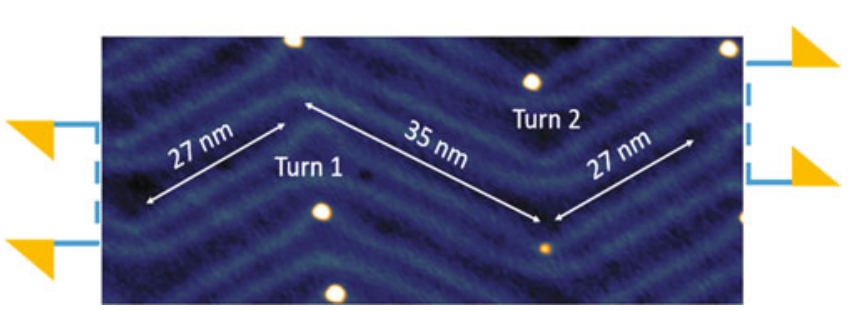

Fig. 2. LT-UHV-STM image of a $75 \mathrm{~nm} \times 35 \mathrm{~nm}$ portion of the $\mathrm{Au}(111)$ surface with its native herringbone reconstruction recorded with scanner 2 on the Toulouse LT-UHV 4-STM instrument. The measured corrugation is $0.023 \mathrm{~nm}$. The race track per team is determined before the start of the race in consultation with the Track Commissioner and all the team drivers. One example of a possible circuit is presented on this STM image with a first straight line of $27 \mathrm{~nm}$ long, a first turn, a long straight line of $35 \mathrm{~nm}$ long, a second turn and finally the second straight $27 \mathrm{~nm}$ long before the arrival line. The width of the track selected here is $4.5 \mathrm{~nm}$ in average. Image conditions: $I=1 \mathrm{nA}$ and $V=1000 \mathrm{mV}$.

Au atoms along a linear portion of the fcc race track [16]. This slalom was effectively constructed in Singapore using a single tip Createc LT-UHV STM instrument for testing [16]. However, it turned out that the construction time from single $\mathrm{Au}$ atom production to STM single atom manipulations is too long in respect to the limited $38 \mathrm{~h}$ of the race imposed by the liquid He cryostat capacity of the LT-UHV 4-STM instrument. The organizers have therefore decided to take benefit of the native turns existing along the rafter due to herringbone reconstruction on the $\mathrm{Au}(111)$ surface, instead of an atomic scale slalom.

The Dresden team was the first team to run on this now official race track which can be defined, as presented in Figure 2, by a first straight line about $27 \mathrm{~nm}$ long, a first right turn, a long straight line of $35 \mathrm{~nm}$ on the fcc domain, a second turn left and finally a second straight $27 \mathrm{~nm}$ long before the arrival line. This makes a $90 \mathrm{~nm}$ long road which can be segmented differently by the teams as soon as their final race track has two turns, three straight lines and a length of about $80 \mathrm{~nm}-90 \mathrm{~nm}$. The departure and the arrival lines can be easily marked by each team during the preparation of their race track using for example their unused molecule-vehicles. The nano-car race commissioner is in charge of certifying the regularity of each race track, so that all of the teams have to run over the same length. The race track in Figure 2 may seem quite short in view of the $38 \mathrm{~h}$ duration of the nano-race event. However, as experienced by the Dresden team during its training, it is long enough for this first edition of the race because of all the atomic scale events that can occur during a run at the atomic scale.

A molecule-vehicle is very sensitive to the atomic scale structure of a race track as presented in Figure 3. On the move and whatever the molecular design is, minimum energy positions of a given molecule-vehicle physisorbed at low temperature is governed by the van der Waals interactions between the molecule-vehicle wheels, paddles or wings and the $\mathrm{Au}(111)$ surface. This determines a landscape of a large number of potential energy

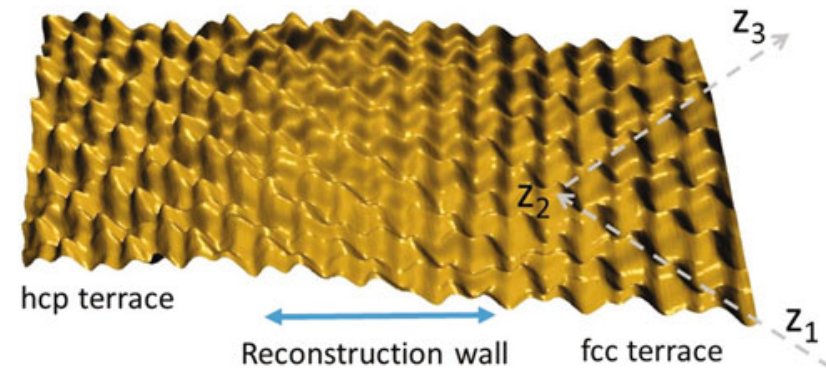

Fig. 3. A pseudo 3D LT-UHV-STM constant current image of the experimental atomic scale structure of the transition between an hcp and an fcc domains recorded on the Figure 2 $\mathrm{Au}(111)$ surface with an atomic corrugation of about $6 \mathrm{pm}$. For a molecule and at low temperature, the reconstruction wall is an efficient slider and the molecule can bounce on it but can also be trapped [18]. The $\mathrm{Z}_{1}-\mathrm{Z}_{2}-\mathrm{Z}_{3}$ path is the trajectory followed by the windmill molecule of the Dresden team when driving in a zig zag manner along a straight fcc race track in a way to avoid the immobilization of the windmill on the central reconstruction wall. The location of the $\mathrm{Z}_{2}$ turning point must be determined with precision (see Sect. 4 for discussion). (Image size: $1.63 \mathrm{~nm} \times 4.96 \mathrm{~nm}, I=300 \mathrm{pA}, V=200 \mathrm{mV}$ recorded on scanner 4 on the Toulouse LT-UHV 4-STM instrument with an exceptional good tip for atomic corrugation on a metal surface).

minima that a given molecule-vehicle will explore on the move. In particular, this landscape is determined by the details of the surface atomic structure of the $\mathrm{Au}(111)$ fcc domain located in between the $0.02 \mathrm{~nm}$ high lateral folding's which constitute the sliders along the race track as presented in Figure 2. More complicated is the energy potential landscape resulting from the atomic structure of the turns along the race track. The atomic-scale details of this turning landscape is also very important to determine per selected turn. A driver must be sure to be able to turn the vehicle as fast as possible, since none of the accepted molecule-vehicles has yet a front or rear rotating link like a mechanical gimbal to control the orientation of the wheels, the paddle or the wing for a safe turn. During its training, the Dresden team has experienced difficulties to negotiate a turn along a $4.5 \mathrm{~nm}$ wide fcc race track.

\section{The Dresden molecule-vehicle on the race track}

The windmill molecule, is a supramolecular assembly on-surface synthesized and made of 4 acetylbiphenyl (ABP) molecules interacting by hydrogen bonding on the $\mathrm{Au}(111)$ surface [12]. An ultra-pure white powder of ABP molecules is first produced from commercially available ABP. Then, a few milligram of this powder is introduced in a small crucible which is mounted on a port of the LT-UHV 4-STM preparation chamber with a controlling valve at its nozzle. Due to the vapor pressure of the ABP substance, molecules will be sublimated and supramolecular assemblies will be formed on the surface without 

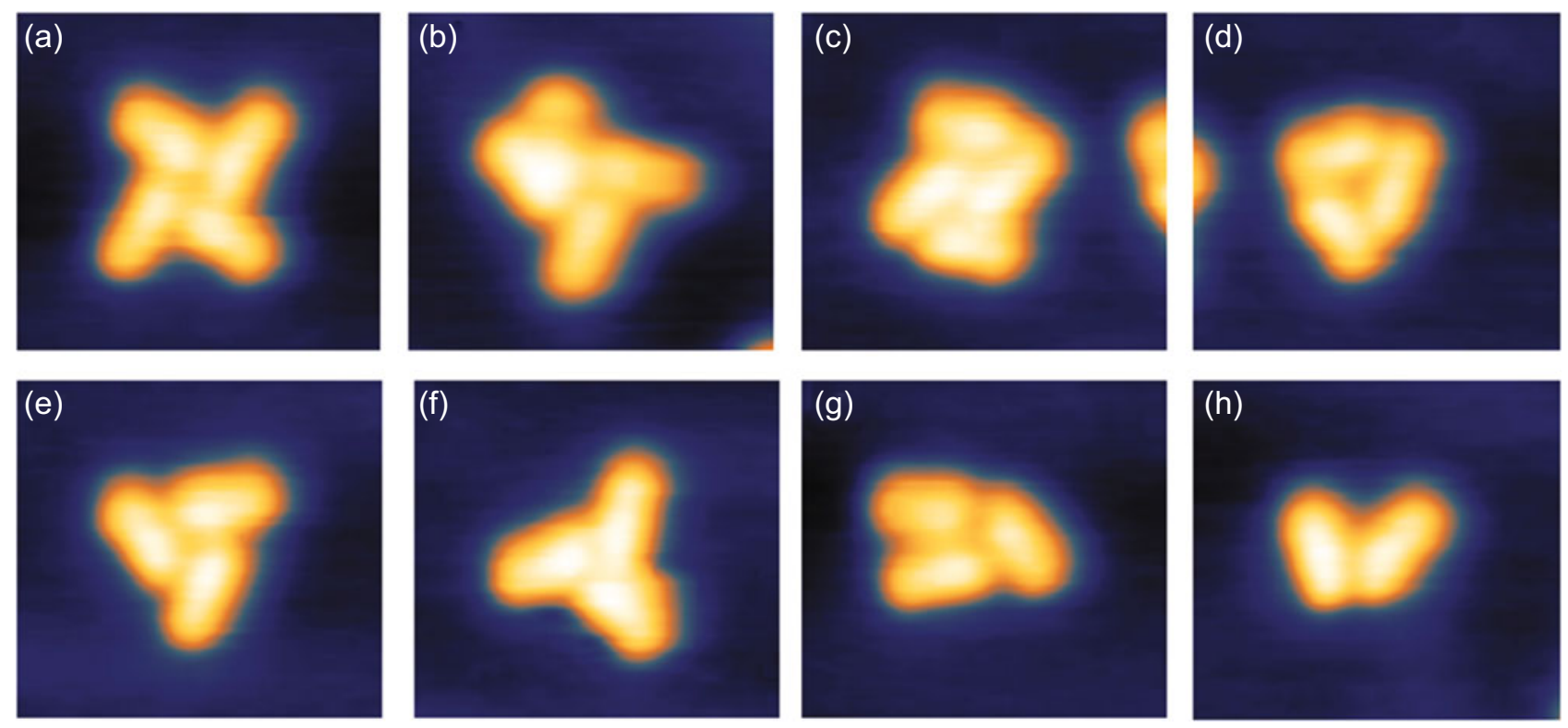

Fig. 4. Different LT-UHV STM constant current images recorded on an $\mathrm{Au}(111)$ surface showing all the different $\mathrm{ABP}$ molecular structures observed in Toulouse on the LT-UHV 4-STM using any of the STM equipped mainly with a vertical tip holder. (a)-(c) are ABP tetramers with (a) the windmill molecule-vehicle registered for the nano-race, (d)-(g) are the often observed ABP trimers when the on-surface synthesis conditions are not perfect and (h) often observed ABP dimers (image size: $5 \mathrm{~nm} \times 4 \mathrm{~nm} ; I=50 \mathrm{pA} ; V=500 \mathrm{mV})$.

heating the crucible. Nevertheless and as compared to the Dresden home prepared windmill molecules from the same ABP powder, it was necessary on the Toulouse instrument to adjust the evaporation time and the crucible temperature because of the different volume of the preparation chamber and the different distance between the port nozzle and the $\mathrm{Au}(111)$ surface in Toulouse and in Dresden. To form windmill structures on the surface, one needs a minimum molecular flux onto the surface. Therefore, the crucible in Toulouse was heated up to $40{ }^{\circ} \mathrm{C}$ during the sublimation. The on-surface synthesis of even more windmill structures was achieved by heating up the $\mathrm{Au}(111)$ surface up to $55^{\circ} \mathrm{C}$ during $300 \mathrm{~s}$ of sublimation. Thereby the diffusion of the molecules is increased and more tetrameric structures can be formed. After the evaporation and heating steps, the $\mathrm{Au}(111)$ surface was transferred along the LT-UHV 4-STM chamber as explained in Section 3 and mounted on the 4-STM head maintained at $4.3 \mathrm{~K}$. Low voltage and low feedback loop currents down to $50 \mathrm{mV}$ and $10 \mathrm{pA}$ were used to image the on-surface synthesized molecular assembly on the $\mathrm{Au}(111)$ surface using any of the 4-STM scanners. The different observed ABP dimers, trimers and tetramers are presented in Figure 4 . With a fine tuning of the evaporation, the heating times and the $\mathrm{Au}(111)$ surface temperature, the Dresden team was able to reproduce its home condition to get $30 \%$ of windmills on the $\mathrm{Au}(111)$ surface with a low submonolayer coverage.

The zoology of dimers, trimers and tetramers was such that the Dresden team took the occasion of its training in Toulouse to play with all those supramolecular structures. Figure $4 \mathrm{e}$ shows an example of the loss of one wing during a run along the race track or at a turn (see Sect. 5).
Tetramer (a) can be transformed into (b) after a voltage pulse of $2.1 \mathrm{~V}$ exceeding a duration of $8 \mathrm{~s}$ and locating the STM tip apex at the center of the tetramer. After another voltage pulse, the reverse phenomenon was also observed, switching back the molecule conformation from (b) to (a). Tetramer (c) was rarely observed and seems to be very robust and needs firstly to be mechanically disordered to reach tetramer (b) conformation. For that purpose, the STM tunneling junction resistance must be largely reduced below $10 \mathrm{M} \Omega$ for the tip apex end atom to enter in a repulsive interaction mode with tetramer (c). Regarding trimers, trimers (d) and (f) were found very stable, and trimers (e) can be converted in trimer (f) also after a $2.1 \mathrm{~V}$ pulse. For completeness, a rarely observed dimer structure (h) is also presented. Among all those on-surface assembled molecular structures formed using ABP monomers, the windmill tetramers have been registered for the nano-car race, as they have shown stable non mechanical manipulation capability, i.e., to be inelastic driven on the $\mathrm{Au}(111)$ surface [12].

Single tip LT-UHV-STM images and inelastic tunneling current manipulation conditions of the windmill tetramers have been extensively studied $[12,14]$. Figure 5 shows a scheme of the on-surface synthesized windmill molecule-vehicle held together via hydrogen bonds, as well as an assembly on the track between the two sliders recorded with a given tip on the LT-UHV 4-STM and one calculated STM image. In the case of the recorded molecule-vehicle, there is no wing aligned with the fcc domain atoms. Therefore an alternated zig zag motion is needed to drive along the track. This motion can be triggered by applying voltage pulses on two different wings of this molecular assembly. 
(a)

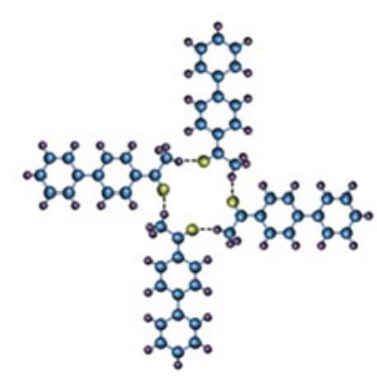

(b)

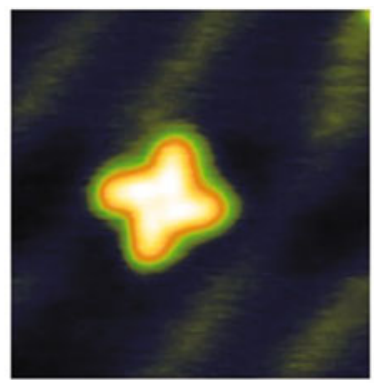

(c)

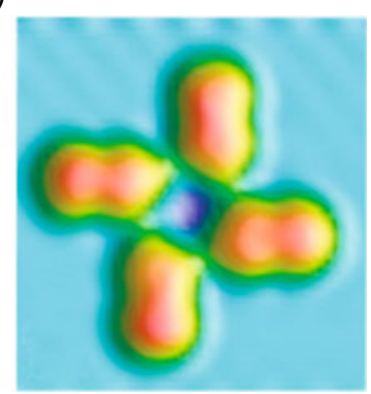

Fig. 5. (a) Atomic structure of a windmill nano-vehicle on $\mathrm{Au}(111)$ with blue: carbon atoms, green: oxygen atoms and purple: hydrogen atoms (dashed lines, the on-surface formed hydrogen bonds). (b) Constant current STM image of a supramolecular assembly well defined on a fcc $\mathrm{Au}(111)$ domain between two rafters (image size: $8 \mathrm{~nm} \times 8 \mathrm{~nm} ; I=0.05 \mathrm{nA} ; V=500 \mathrm{mV}$ ). (c) The corresponding calculated STM constant current image $(3.8 \mathrm{~nm} \times 3.8 \mathrm{~nm})$ (see Ref. [12] for more details).

To determine the values for the voltage pulses for a translation in between the $\mathrm{Au}(111)$ sliders or to perform a rotation for passing a turn, one needs to master the scanning tunneling spectrum (STS) $\mathrm{d} I / \mathrm{d} V$ of the molecular tetrameric assembly as recalled in Figure 6 . At positive bias voltage, an electronic resonance at $+2.5 \mathrm{~V}$ is observed and one needs to tunnel into this molecular resonance to induce the movement. Due to the broadness of this resonance, a voltage of $+1.8 \mathrm{~V}$ is already enough to tunnel into the resonance and to trigger the motion (either translation or rotation as described in [12]). For higher voltage values exceeding $+2.5 \mathrm{~V}$, hydrogen-bonds break and the windmill molecule-vehicle is destroyed.

Let us now consider a single windmill molecule on the $\mathrm{Au}(111)$ surface well aligned on the $\mathrm{Z}_{1}-\mathrm{Z}_{2}$ direction defined in Figure 3. On the electronic ground state potential energy surface of the quantum system formed by the windmill and the $\mathrm{Au}(111)$ surface, the $\mathrm{Z}_{1}-\mathrm{Z}_{2}$ direction can be considered as the motion reaction path, where each stable position is separated from the next one by about $0.3 \mathrm{~nm}$. It is not exactly $0.2884 \mathrm{~nm}$ because of the two lateral wings. In a flat portion of the fcc domain and at $30^{\circ}$ relative to the slider orientation, there is about 10-12 such minima along this $\mathrm{Z}_{1}-\mathrm{Z}_{2}$ direction before the front wing and the left lateral wing of the windmill start to be mechanically deformed by the first atomic row of this slider. Without a mechanical push, the only way to pass from one minimum to the next along the $\mathrm{Z}_{1}-\mathrm{Z}_{2}$ motion reaction path is to reach the first low lying excited states of the front wing. This was performed by the Dresden team by pulsing the bias voltage, but remaining at a low feedback tunneling intensity to avoid any destruction of the windmill. In this case, a resonant tunneling phenomenon occurs through those excited states. Here, the electrons are mainly transferred elastically through the excited ABP wing. However, about $0.01 \%$ of those electrons are not transferred elastically. Since even for a $10 \mathrm{pA}$ of current, there are still millions of electrons transferred per second through ABP and this leads to an increase of its reached excited state vibrational energy [19]. Normally, this energy excess is redistributed inside the molecular structure and then to the surface. But for the windmill molecular structure and certainly due to the relative

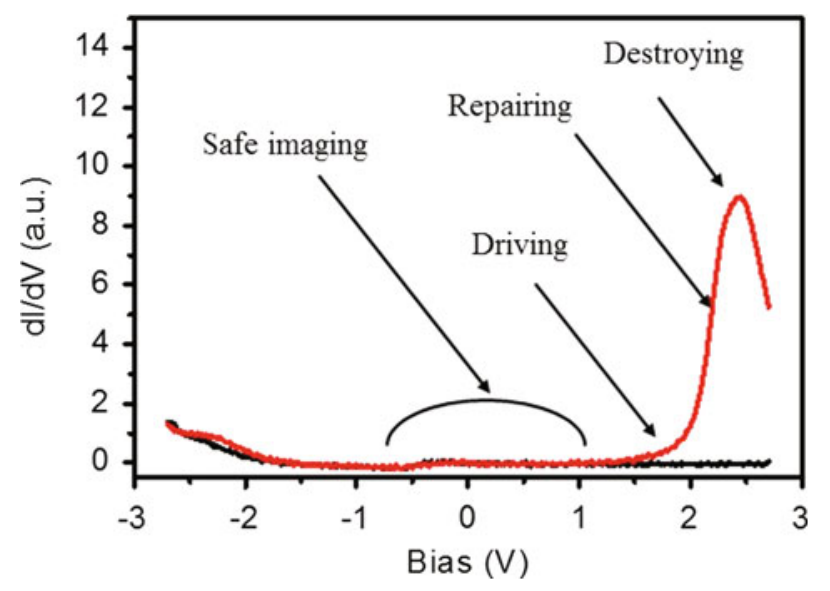

Fig. 6. $\mathrm{d} I / \mathrm{d} V$ LT-UHV-STM spectra (adapted from Ref. [12]) recorded on the bare $\mathrm{Au}(111)$-surface (black) and on one wing of the windmill molecule (red). The different critical voltage ranges for safe imaging, safe driving, destruction threshold at the first electronic resonance and repairing bias voltages are also indicated.

independence of the electronic structure of the $4 \mathrm{ABP}$ in the tetramer, a part of this vibrational energy is consumed by the windmill molecular structure to move from one minimum energy position on the surface to the next in direction of the excited $\mathrm{ABP}$ and with no destruction of the windmill when the voltage pulse amplitude is well tuned. For this purpose, one has also to wait long enough for the vibrational energy to accumulate in the reached excited states for the reaction path to develop along the $\mathrm{Z}_{1}-\mathrm{Z}_{2}$ direction on the excited states potential energy manifolds. Experimentally, the determined minimum voltage pulse rate is between 0.1 and $1 \mathrm{~Hz}$ [12]. In effect, one has to wait a little bit longer for the voltage pulse to be well shaped in time because of the large STM tunneling resistance in regards with the few $\mathrm{pF}$ stray capacitance of the STM junction. For the peculiar intramolecular structure of the windmill, this inelastic process also implies that the succession of minima in the $\mathrm{Z}_{1}-\mathrm{Z}_{2}$ direction on the potential energy manifold of the excited states might not be exactly aligned with the corresponding maxima of the 

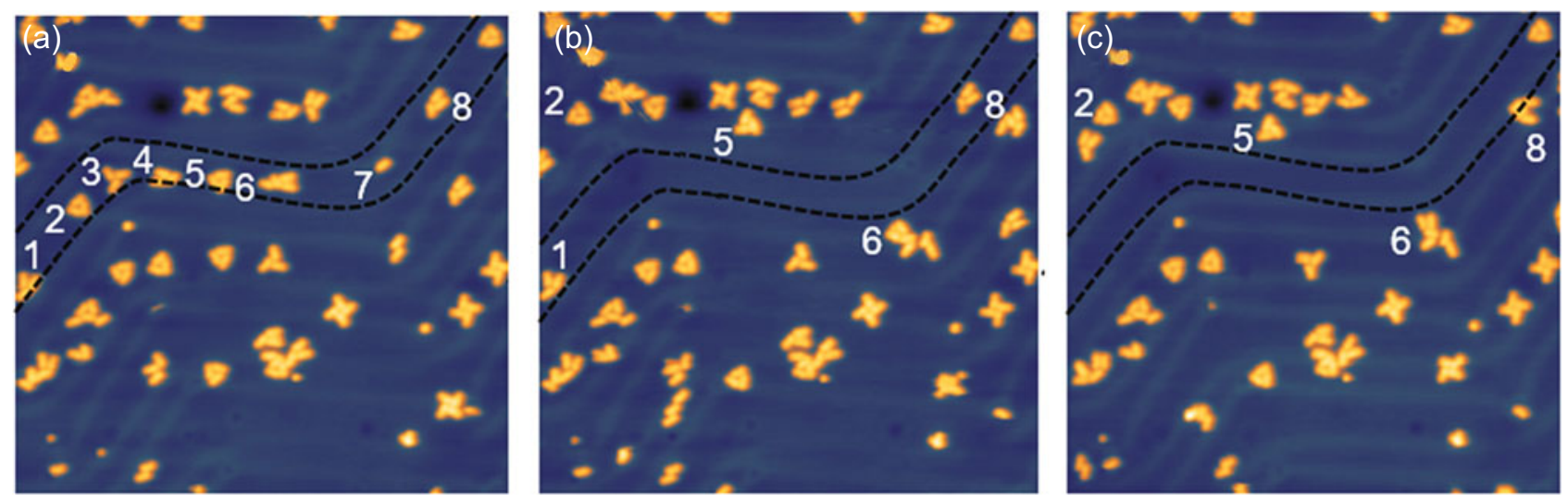

Fig. 7. LT-UHV STM image recorded with scanner 2 on the Toulouse LT-UHV 4-STM of the Dresden selected track (a) before cleaning and (b) after scanning twice over the area between molecule two and seven in a pushing molecular manipulation mode using $I=2 \mathrm{nA}, V=50 \mathrm{mV}$ and (c) after manipulating molecule one and eight (manipulation parameter: $I=1.0 \mathrm{nA} ; V=2.0 \mathrm{~V}$; $t=5.0 \mathrm{~s}$ ) (image sizes: $50 \mathrm{~nm} \times 50 \mathrm{~nm} ; I=20 \mathrm{pA} ; V=500 \mathrm{mV}$ ).

windmill ground state potential energy surface also in the $\mathrm{Z}_{1}-\mathrm{Z}_{2}$ direction [4]. It is this shift in energy that provides to the windmill molecular structure an internal quantum motor [4], even if this motor has not the classically distinguishable form of a molecular motor [8]. The Dresden team has also observed that two jumps of $0.3 \mathrm{~nm}$ can occur under a single voltage pulse. In this case, the excited state energy relaxation is slowed down enough, so that the reaction path can directly reach the second available minimum on the reached excited states manifold.

To respect the nano-race rules [16] and before its first run with a windmill, the Dresden team had also selected a given race track on the $\mathrm{Au}(111)$ surface with two turns separated by about $35 \mathrm{~nm}$ of a straight path. Natively, after the ABP monomers evaporation and after a successful windmill on-surface synthesis, the windmills are distributed randomly all over the herringbone reconstructions. However, the race track must not be selected at random. Firstly, it had a minimum of windmills on it to speed up its cleaning. Secondly, Dresden had payed attention of the lateral width of each turn in relation with the overall van der Waals width of a windmill. In this way, a windmill molecule will not to be trapped by the slider when reaching a turn. For this purpose, Dresden had selected the race track presented in Figure 7a. Furthermore, at this position no defects have been found at the elbow sites, which would be a barrier for the molecule at this position. The cleaning started step by step, STM manipulating one after the others of the windmill molecules located along the selected race track. Here, the molecular manipulation mode can be a pushing mode [20], while respecting the nano-race rules [16]. The complete cleaning of the Figure 7 a race track by Dresden took about $2 \mathrm{~h}$, i.e., well below the $6 \mathrm{~h}$ limit fixed by the nanorace organizers [16]. The final result is presented in Figure $7 \mathrm{c}$. One interest of this initial race track preparation step is that each team will be able to park supplementary molecule-vehicles nearby its selected track. In case of an accident occurring on the run and if it is not possible to repair this molecule on the spot using the
STM tip (see Sect. 5), it is permitted to bring a new intact molecule-vehicle at the location of the accident to continue the nano-race. The macroscopic Formula E competition is doing the same to not lose time in exchanging the batteries. After this full cleaning step, the Dresden team left one windmill on track and was ready for running sessions.

\section{Driving the Dresden molecule-vehicle on the $\mathrm{Au}\left(\begin{array}{lll}1 & 1 & 1\end{array}\right)$ reconstruction}

Starting from what is known about the windmill translation and rotation ability by tunneling inelastic effect, the first run was expected to be quite easy. This was not the case, since the initial adsorption position of a given windmill is not always the best orientation to start a run. The Dresden driver had first to be sure that its molecule is oriented with a wing exactly $45^{\circ}$ to the reconstruction wall. Then, the driver can go for a run by systematically selecting the wing pointing in the direction of the movement. In this case and in average, a given voltage pulse of about $8 \mathrm{~s}$ duration is able to displace the molecule by a step of $0.3 \mathrm{~nm}$ and sometimes by a step of $0.6 \mathrm{~nm}$. The interesting point here is that in case of a move of $0.3 \mathrm{~nm}$, there is normally no danger for the front wing to reach the border of the reconstruction (slider) and the driver can avoid this by pulsing on the other wing to move again forward and toward the opposite reconstruction wall. However, in case of a $0.6 \mathrm{~nm}$ move forward, it may happen that the front wing jump on the reconstruction wall as presented in Figure 8.

In that case, the windmill is often stuck in the configuration with no possibility to move again. We have interpreted this first surprise by the fact that the torsion of this front wheel up to about $0.03 \mathrm{~nm}$ by reaching the reconstruction side wall is deforming the potential energy manifold of the windmill in such a way that the potential energy barrier characteristic of the $0.3 \mathrm{~nm}$ linear motion is increased by this distortion. The corresponding inelastic energy delivered during the $8 \mathrm{~s}$ voltage pulse is therefore 
(a)

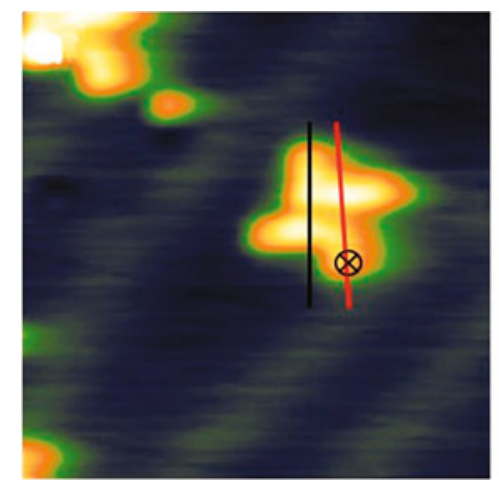

(b)

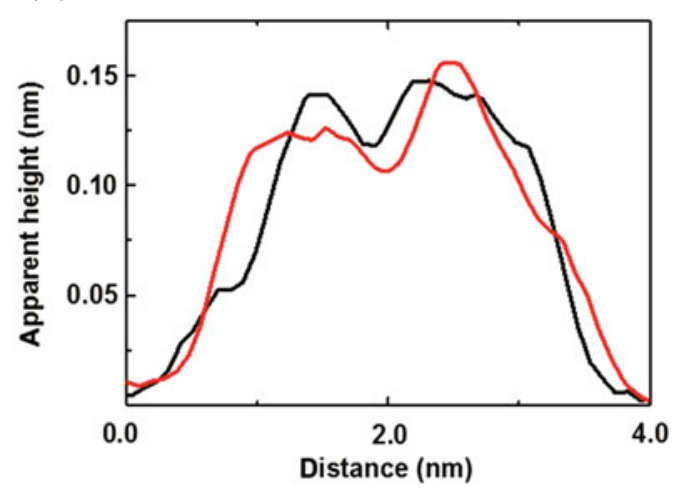

Fig. 8. (a) A windmill molecule pinned at the position marked with the circled cross (image size: $12 \mathrm{~nm} \times 12 \mathrm{~nm}$; $I=0.01 \mathrm{nA} ; V=500 \mathrm{mV}$ ) and (b) line scans over the molecular assembly taken along the black and the red line presented in (a). The pinned molecule having an apparent height of $0.12 \mathrm{~nm}$ instead of $0.15 \mathrm{~nm}$ for the other molecules in the assembly.

dissipated towards all the internal degrees of freedom of the windmill and then is dissipated through the $\mathrm{Au}(111)$ surface. If this happens, the Dresden pilot found a solution: by pulsing with a larger voltage on another wing of this molecule, one can destroy the tetramer and recover a trimer + monomer configuration (see Fig. $4 \mathrm{~b}$ ). In that case, another pulse can reassemble the windmill tetramer with a good chance to have now all the wings out of the reconstruction wall due to a rotation of about $20^{\circ}$ of the reconstructed windmill structure (see Sect. 5 for more details).

Aside this trapping problem at the border of the reconstruction, the driving along a straight portion of the reconstruction can be performed by zig zagging between the two reconstruction walls along the fcc domain, which is about $4.5 \mathrm{~nm}$ in width as described above. Ideally and by step of $0.3 \mathrm{~nm}$ in $8 \mathrm{~s}$, the maximum time for a run of about $100 \mathrm{~nm}$ would be about $45 \mathrm{~min}$. In reality, it has been experienced during the Dresden training session that a driver needs to record at least one STM image after each pulse to certify the new position of the molecule-vehicle. A good quality STM image for the next pulse location takes about $3 \mathrm{~min}$ in a standard $10 \mathrm{pA}$ set up condition. This leads us to a $100 \mathrm{~nm}$ drive in $16 \mathrm{~h}$. Of course, a good and attentive driver will be able to avoid some images by looking carefully at the variation of the tunneling current vs. time during the pulse duration. The current variation during this short time window can signal a move of the molecule. For the windmill, a $0.3 \mathrm{~nm}$ move in one direction following the $\mathrm{Au}$ atom row is almost delivering no current variation since as presented in Figures 5 and 9, the STM contrast variation along a given wing is very small during such a move. The short $5 \mathrm{~nm}$ run presented in Figure 9 is the result of a $57 \mathrm{~min}$ drive cumulating 19 pulses of $8 \mathrm{~s}$ each with the corresponding 19 constant current images of 3 min each. Following this driving speed a straight line of $100 \mathrm{~nm}$ would have required a drive of $24 \mathrm{~h}$ still under the maximum time allowed for the Toulouse race. As during the race four driver need to manipulate the molecules at the same time, in this training session we successfully manipulated molecules with one tip and imaged at the same time with another tip another quadrant of the gold pastille without influencing each other.

After a long zig zagging drive, it was more difficult for the windmill to turn. As presented in Section 2, there are generally large and narrow turns along a large $4.5 \mathrm{~nm}$ track, at the end of a straight line of about $30 \mathrm{~nm}$ in length. Due to the underneath fcc atomic structure of the surface of the track and due to the fact that the windmill molecule can be driven safely only along a fcc atomic row, the windmill must be rotated at the end of a straight track to be aligned on the atomic rows of the turn and then along the fcc atomic rows of the next straight fcc part after the turn. To turn, the windmill requires to alternatively apply voltage pulses on the front driving wing and also on another wing. Of course, this succession of $0.3 \mathrm{~nm}$ translation and rotation is very delicate since it is virtually impossible to image at the same time the windmill molecule and the exact position on the Au atoms at a turn. Nevertheless, it is known that at the elbow site one single $\mathrm{Au}$ atom is missing and therefore, it is for example a preferred nucleation center for metals [21]. Also molecules are known to likely occupy this turning point [22], which has been shown to be useful for other experiments. As the molecules do not like to move, when attached to the elbow-sites, they are used for example for direct imaging of molecular orbitals [23]. During the Dresden training session, we have experienced the adsorption of the molecules on the elbows during the driving along the turn. This became a problem as the molecule was very often blocked. To overcome this problem of the nano-car stacking at a turn, the Dresden team had decided to apply stronger voltage pulses on the windmill, trying to pass over the turn via the reconstruction wall, even if there was a risk to destroy the windmill structure. This method worked a few times. However, in the vast majority of the attempts, the molecular assembly did not move at all and at a certain point one wing of the windmill was lost. This means that the adsorption energy at such a defect site is reduced drastically and therefore a further movement by applying voltage pulses is rather difficult. Each team needs to take this into account, as they will have the same 

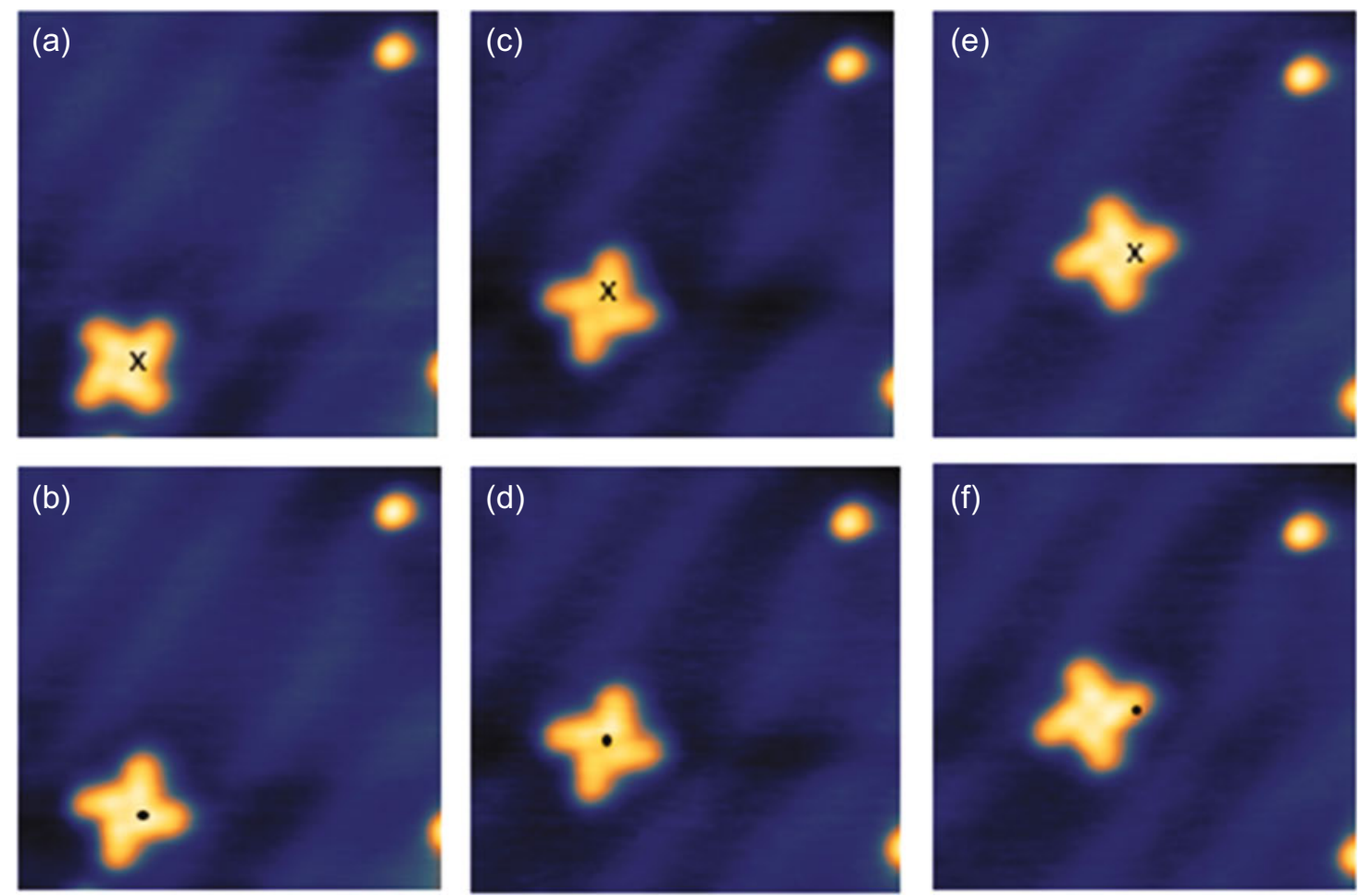

Fig. 9. Typical events of motion after voltage pulses are presented in figures from (a) to (f). The cross marks the position of the voltage pulse before and the dot is marking the position of the voltage pulse after the motion of the molecule. From (a) to (b) a rotation of the assembly took place. From (c) to (d), the molecule was moved in the direction to the STM tip. From (e) to (f) the molecule was moved away from the STM tip (image size $10.5 \mathrm{~nm} \times 10.5 \mathrm{~nm} ; I=0.05 \mathrm{nA}$; $V=500 \mathrm{mV}$; manipulation parameter: $I=1.0 \mathrm{nA} ; V=2.3 \mathrm{~V}$; driving pulse time $t=8.0 \mathrm{~s}$, recording duration of each presented (a)-(f) STM images: $3 \min )$.

problems. On the other hand, the destruction of the assembly lead to the discovery that one can repair a windmill on the spot as discussed in the next section. Such a training session will certainly encourage the teams to precisely map out their own selected two turns with an atomic precision before starting the race.

\section{Accidents and repairs}

During the training session in Toulouse, different types of accidents occurred to the Dresden windmill molecule on the race track. The most frequent one was the rotation of one wing with a formation of a trimer as presented in Figure 10, plus the 4th ABP molecule going in between two wings of this newly formed trimer. This happened mainly when the driving voltage pulse was accidentally too large or when intentionally the pulse was larger to de-block a windmill molecule which had gone too far on a reconstruction wall or to pass over a narrow turn, as explained at the end of the last section. Here, we will describe some of the accidents, which have occurred during the training and how the Dresden team succeeded to repair on the spot a few of the windmills to continue the run. For all those cases and working $90 \%$ of the time, the solution found by the Dresden team was to further apply voltage pulses, generally in the center of the molecule, with a bias voltage around $2.1 \mathrm{~V}$. In that case, the windmill molecule was generally recovering its tetramer windmill shape with apparently no damage but with a rotation. One advantage of the Dresden molecule-vehicle for the competition is therefore to be repairable on the race track.

A further annoying accident happened when, during a straight drive, the windmill molecule suddenly jumped to the tip and reappeared in the next STM image at another location on the surface. We have not yet an explanation of this phenomenon, which fortunately is quite rare. One interpretation is that after a large number of bias voltage pulses, the tip apex end atomic structure is re-organized. This creates a different inhomogeneous electric field pattern at the end of the tip, which can attract one windmill on the tip apex.

There is also another interesting cumulative effect. When the windmill is stacked at a turn or at a reconstruction edge, it happens that a too large number of pulses destroys the molecular structure. This is due to the fact that when stacked, vibrational energy accumulates in the excited states and is passing over the reaction barrier for detachment of some wings from the windmill tetramer molecular structure. Fortunately, this phenomenon never happened during a good run where at least 50 voltage pulses could be cumulated without any destruction of the windmill molecule-vehicle. 

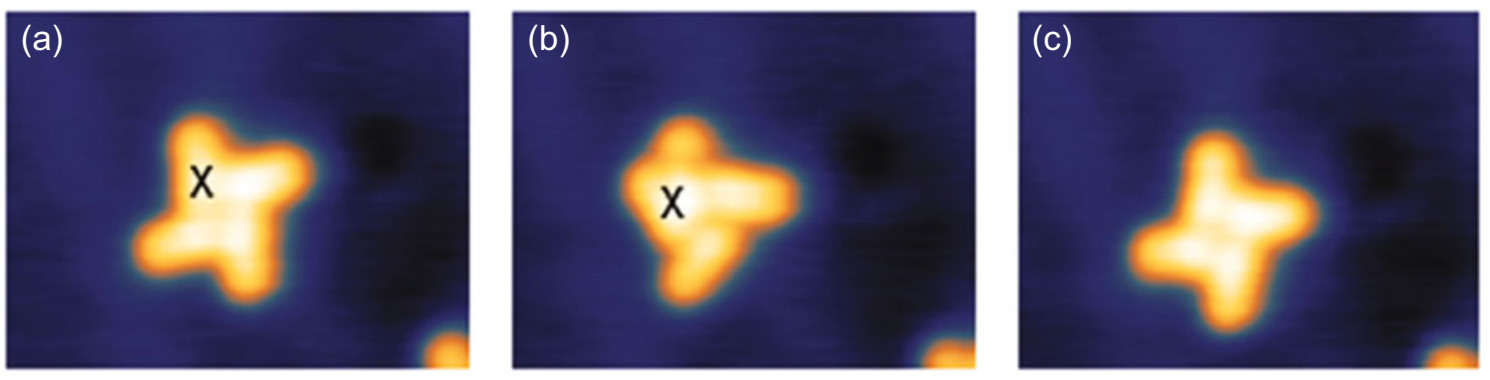

Fig. 10. (a) An intact molecule-vehicle attempted to be manipulated by applying a voltage at the position marked by the cross for moving the assembly (manipulation parameter: $I=1.1 \mathrm{nA} ; V=2.3 \mathrm{~V} ; t=8 \mathrm{~s}$ ) and (b) the same molecule reorganized after this voltage pulse, another voltage pulse was applied on the molecular assembly, the position is marked with the cross (manipulation parameter: $I=0.5 \mathrm{nA} ; V=2.0 \mathrm{~V}$ ) (image sizes: $8.4 \mathrm{~nm} \times 5.4 \mathrm{~nm} ; I=0.05 \mathrm{nA} ; V=500 \mathrm{mV}$ ). (c) The final conformation of the windmill molecule after the bias voltage pulse in (b). The only difference between (a) and (c) is a small rotation of the molecule of about $20^{\circ}$.

\section{Conclusion}

In summary, we have presented the first training of the Dresden Team on the LT-UHV 4-STM in Toulouse in order to prepare the first international nano-car race. During this training, the preparation of the $\mathrm{Au}(111)$ surface was successfully performed over the complete gold pastille, and a race track was defined taking into account the characteristics of the $\mathrm{Au}(111)$ herringbone reconstruction. The Dresden windmill molecule-vehicle was successfully on-surface synthetized and imaged by STM by any of the four tips of the LT-UHV 4-STM instrument. The windmill was controllably driven by inelastic electronic tunneling effects. The Dresden team has learnt how to drive its windmill vehicle along a herringbone track and has developed new driving strategies to turn along a given $\mathrm{Au}(111)$ fcc track. Moreover, some accidents had occurred during the training, which gave the Dresden team the possibility to learn how to repair on the spot the windmill vehicle and to continue the run.

We thank the Midi-Pyrénées "Contrat de Plan Etat Region Campus G. Dupouy" (2007-2014) for the complete financial support for the LT-UHV 4-STM machine and particularly the constant support from the Region Midi-Pyrenees, the Toulouse Metropole, the FEDER and the CNRS. We thank our nano-car race sponsors: ScientaOmicron, Peugeot PSA, Toyota, Air Liquide, The Foundation "Maison de la Chimie", ACTIA Toulouse, NanoScience GSO, Nature Nanotechnology and EDF, CASDEN Banque Populaire and MGEN for continuous equipment's, software, financial supports and encouragements. The work was also funded by the MANA-NIMS Japanese MEXT WPI program through the Toulouse MANA Satellite. Support by the German Excellence Initiative via the Cluster of Excellence EXC1056 "Center for Advancing Electronics Dresden (cfaed)" and the International Helmholtz Research School Nanonet is gratefully acknowledged.

\section{References}

1. C. Joachim, G. Rapenne, ACS Nano 7, 11 (2013)

2. B.C. Stipe, M.A. Rezaei, W. Ho, Science 279, 1907 (1998)
3. S.W. Hla, L. Bartels, G. Meyer, K.H. Rieder, Phys. Rev. Lett. 85, 2777 (2000)

4. J. Echevaria, S. Monturet, C. Joachim, Nanoscale 6, 2793 (2014)

5. J. Yang et al., Eur. Phys. J. Appl. Phys. 73, 10702 (2016)

6. Discussions about Molecule-Trucks During the NATO ARW on Nano-Age Mechanics (Loch Lomond, Scotland, 1995)

7. M.T. Michalewicz, Ann. Improb. Res. 4, 1 (1998)

8. G. Vives, J.M. Tour, Acc. Chem. Res. 42, 473 (2009)

9. Y. Shirai, K. Minami, W. Nakanishi, Y. Yonamine, C. Joachim, K. Ariga, Jpn J. Appl. Phys. 55, 1102A2 (2016)

10. T. Kudernac, N. Ruangsupapichat, M. Parschau, B. Maciá, N. Katsonis, S.R. Harutyunyan, K.H. Ernst, B.L. Feringa, Nature 479, 208 (2011)

11. R. Joseph, A. Nkrumah, R.J. Clark, E.J. Masson, J. Am. Chem. Soc. 136, 6602 (2014)

12. A. Nickel, R. Ohmann, J. Meyer, M. Grisolia, C. Joachim, F. Moresco, G. Cuniberti, ACS Nano 7, 191 (2013)

13. E.C. Constable, C.E. Housecroft, J.R. Price, J.A. Zampese, Cryst. Eng. Commun. 12, 3163 (2010)

14. R. Ohmann, J. Meyer, A. Nickel, J. Echevaria, C. Joachim, F. Moresco, G. Cuniberti, ACS Nano 9, 8394 (2015)

15. Le Petit Journal, supplément illustré, $\mathrm{n}^{\circ} 194 \mathrm{du} 6$ aout 1894

16. http://www.cemes.fr/Molecule-car-Race and see also http://nanocar-race.cnrs.fr/

17. F. Hanke, J. Björk, Phys. Rev. B 87, 235422 (2013)

18. C. Manzano, W.H. Soe, H.S.J. Wong, F. Ample, A. Gourdon, N. Chandrasekhar, C. Joachim, Nat. Mater. 8, 576 (2009)

19. S. Monturet, M. Kepenenkian, N. Lorente, C. Joachim, Chem. Phys. Lett. 573, 1 (2013)

20. T.A. Jung, R.R. Schlittler, J.K. Gimzewski, H. Tang, C. Joachim, Science 271, 181 (1996)

21. J.A. Meyer, I.D. Baikie, E. Kopatzkie, R.J. Behm, Surf. Sci. Lett. 365, L647 (1996)

22. P. Maksymovych, D.C. Sorescu, D. Dougherty, J.T. Yates Jr., J. Phys. Chem. B 109, 22463 (2005)

23. W.-H. Soe, C. Manzano, A.D. Sarkar, N. Chandrasekhar, C. Joachim, Phys. Rev. Lett. 102, 176102 (2009) 